\title{
Picturing diversity in the ATLAS collaboration
}

\author{
Silvia Biondi* \\ University "Alma Mater Studiorum" \& INFN - Bologna, Italy \\ E-mail: silvia.biondiecern.ch
}

\begin{abstract}
With over 3000 members from 178 institutes, the ATLAS Collaboration is naturally diverse. Capturing this diversity through pictures can be a challenge. Photography is a powerful tool, allowing us to reveal the faces behind a story and give the public the unique opportunity to understand and appreciate the human aspects of ATLAS's scientific research. The role of photographs in portraying the diversity of the ATLAS Collaboration on various communication platforms will be described. Their impact will be examined, with focus on engagement of new audiences.
\end{abstract}

EPS-HEP 2017, European Physical Society conference on High Energy Physics 5-12 July 2017

Venice, Italy

${ }^{*}$ on behalf of the ATLAS Collaboration 


\section{Introduction}

The ATLAS Collaboration [1] is composed of over 3000 members from 178 institutes, as shown in Figure 1 (left). For this reason, diversity is the reality of all the scientists working in this environment. Every day, at CERN, thousands of people, with different genders, traditions and ethnicities, meet each other to collaborate and reach a common goal.

The ATLAS collaboration has set up a Diversity task force which studied the collaboration composition, in terms of gender, ages, role and geography [2]. Figure 1 shows the ATLAS collaboration members nationalities: in blue countries where some institutes belong to the ATLAS collaboration, in green countries where there are not such institutes.

The term diversity can be understood differently by each of the collaboration members, from the recognition of individual differences such as religious beliefs and sexual orientation to well studied issues such as gender composition (largely handled by the Science Europe as well [4]). In this wide topic, four dimensions have been identified by the CERN organisation policy, as shown in Figure 1: nationality and culture, profession, age and generation, gender.
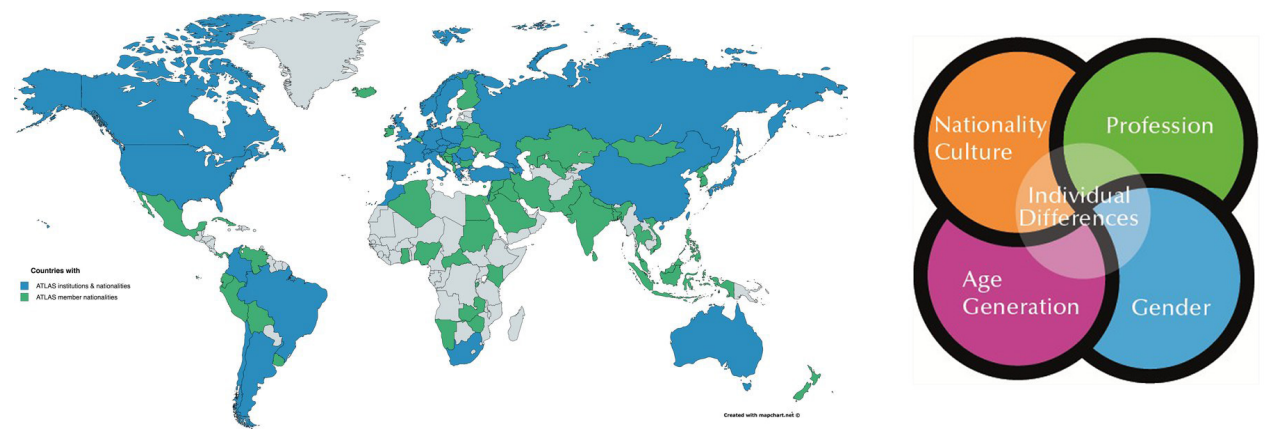

Figure 1: on the left, ATLAS collaboration map [2]. On the right, the CERN schema representing the four dimensions of diversity [3].

Having diversity in mind is extremely important for the groups handling communication and outreach. Although it is part of a scientist's daily life, stereotypes prevent the public from seeing it. For this reason it is worth showing it as much as possible. Also because scientists have their own biases, taking a wide range of pictures and choosing the right messages is mandatory to increase awareness.

\section{The ATLAS Outreach Group: tools and strategy}

The ATLAS outreach effort is shared by all the members of the collaboration who choose to contribute with local exhibitions, events or public talks, guides for visits or virtual visits, etc; but a specific core group is in charge with the development of web platforms, communication and educational content and strategies to support and facilitate these activities.

First of all, the public web site [5] offers a comprehensive space for all links and resources. News items describe the most important events in the life of the collaboration, and are kept up to date. Physics briefings describe the scientific results in a language that can be understood by a wider science and student community. Blogs show the human side of science and are extremely helpful in the public engagement. 
Social media such as Facebook, Twitter and Instagram are used to engage a wider and younger public, eager to learn what science is being done at CERN and how it is being done. Through them, the material posted on the web site can be shared and re-shared. The response level and reactions from the public can be monitored by the core team.

Modern communication relies a lot on multimedia: pictures, videos, animations enhancing the content across platforms. This material can also be used in public (local or remote) talks and events, and is extremely helpful in public engagement. One often says that "a picture is worth a 1000 words".

\section{Picturing people: diversity dimensions}

Photography is a single point of view to catch infinite others and to describe diversity. Through it, it is possible to get to know people behind the story and to tell it by images.

Scientists often work with computers, and picturing them at work can easily become repetitive or artificial. However, one can take advantage of events organized by the ATLAS collaboration, conferences and specific projects such as students programs, internships or awards. A few recent examples are given below.

A well known event is the Summer Student Program that allows young students from many different countries to enjoy and experience the CERN environment and the physicist's daily job. Thanks to this project, different nationalities, genders and backgrounds meet together to work and collaborate for a common aim, as shown in Figure 2. It is crucial to document this succesful CERN project because it demonstrates the diversity of nationalities and cultures.
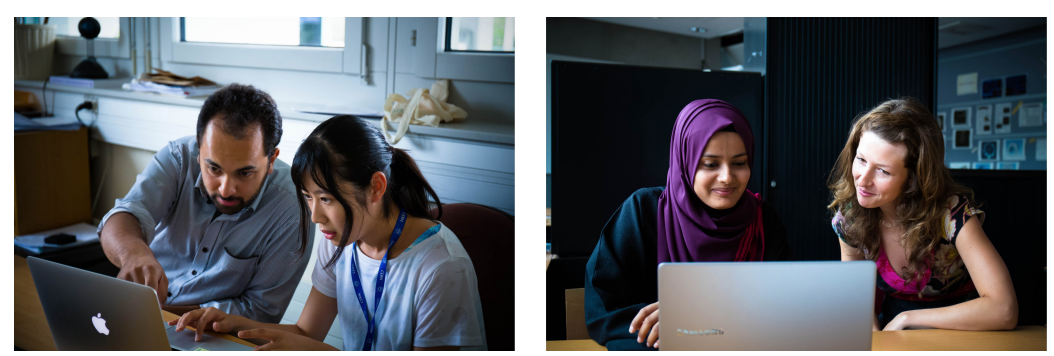

Figure 2: Students from the Summer Student Program and their supervisors working together in CERN offices [6].

Grants ceremonies allow people to meet different and interesting scientists from all over the world and many ATLAS awards events were followed and documented by the outreach group over the years $([7,8,9])$. These ceremonies allow to picture the diversity dimension of ages and generations. Other notable events used for this purpose are the conferences, where different ages and generations present posters and give talks. Moreover, there is always a very high participation of people from many countries. It is, indeed, a perfect occasion for catching these interesting mixtures and giving visibility to youth, Figure 3.

The imbalance of the gender dimension is discussed in the scientific community, in the hope to kill the myth of women being uninterested in science and even not capable of doing science at all. The ATLAS collaboration supported a photographic project on the occasion of the Woman's day, in 

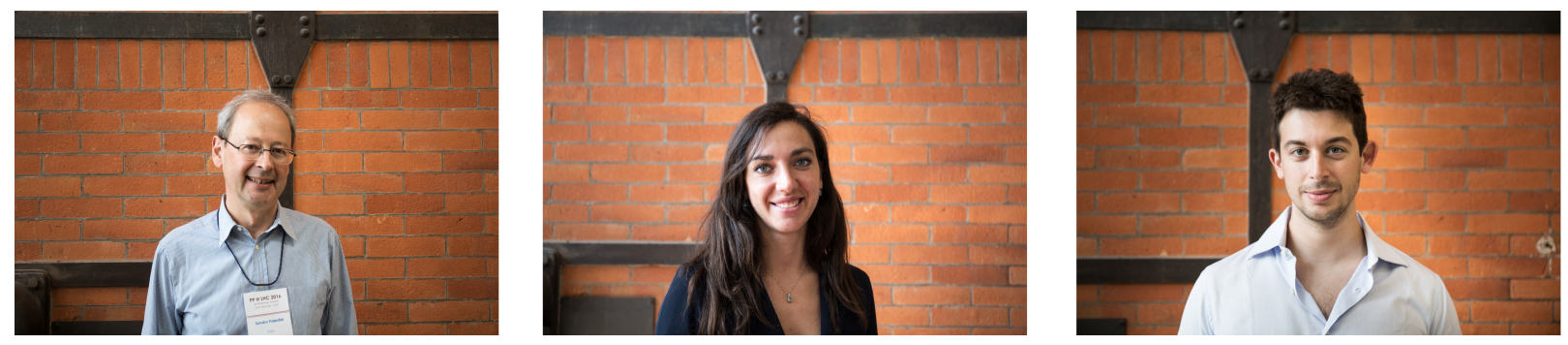

Figure 3: Different gender and generation speakers at the Italian conference LHCpp, in Pisa, 2016 [10].

2016. Many women from different countries and covering different jobs have been photographed and interviewed to tell their own stories, Figure 4. This kind of project allows us to show to girls and women, willing to enter the field, the range of important and challenging responsibilities covered by women.
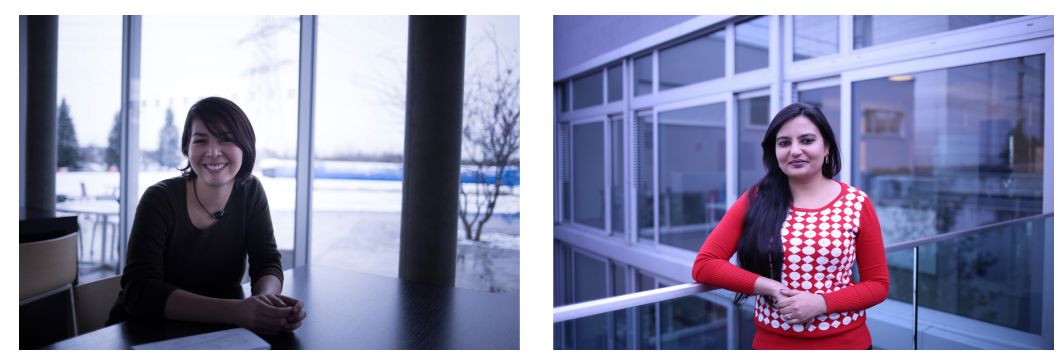

Figure 4: Women of the ATLAS Collaboration, covering different roles and doing different jobs inside the collaboration, sharing thier stories and experiences in occasion of the Woman's Day 2016 [11].

The awards events and ceremonies allow us to illustrate and promote the diversity of professions and skills involved in experimental physics: physicists, engineers, technicians, computing scientists and administrators, Figure 5. Except for direct intervention on the detector, it is not always easy to illustrate which kind of task people are responsible for. For this reason, story telling, interviews and sometime videos are needed. Figuring out how many different jobs a person can do for a common goal helps to highlight, both internally and for the public, the strong collaboration spirit inside the community.
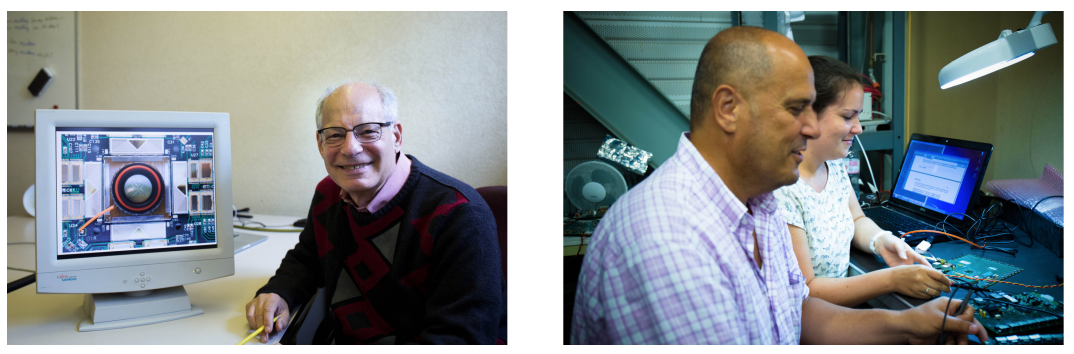

Figure 5: On the left, Alan Litke explaining the invention of a device that records the neural activity of retinal cells [12]. On the right, a student and her supervisor from the Summer Student Program of 2016, in their laboratory at CERN [6]. 


\section{Conclusions}

Illustrating diversity is not limited to specific events or dates such as Woman's day, it has to be considered continuously when carrying out communication. Through images, one must not reinforce the stereotypical view of physicists, but showing the naturally diverse group of people that make up the collaboration.

In this context, every blog post, news piece, social media post and image is a venue to showcase the diverse collaboration. For this purpose, the strong impact of pictures is well known and can be monitored by the ATLAS outreach group toolkit. It has proven to be worth developing.

\section{References}

[1] The ATLAS Collaboration, The ATLAS Experiment at the CERN Large Hadron Collider, JINST 3 (2008) S08003.

[2] The ATLAS Collaboration, Studies related to gender and geographic diversity in the ATLAS Collaboration, ATL-GEN-PUB-2016-001.

[3] Diversity at CERN official web site, http://diversity.web.cern.ch/scope/dimensions-diversity-policy .

[4] Science Europe, Practical Guide to Improving Gender Equality in Research Organisations, https://www.scienceeurope.org/.

[5] The ATLAS Collaboration public web site, https://atlas.cern .

[6] Biondi S., ATLAS summer students and supervisors 2016, ATLAS-PHOTO-2016-015.

[7] Biondi S., ATLAS PhD Grant recipients 2017, ATLAS-PHOTO-2017-005.

[8] Biondi S., ATLAS Thesis Awards 2015, OPEN-PHO-LIFE-2016-001.

[9] Biondi S., ATLAS Thesis Awards 2016, ATLAS-PHOTO-2017-007.

[10] Biondi S. and Franchini M., Spring celebrations in Pisa as the LHC restarts, https://atlas.cern/updates/atlas-blog/spring-celebrations-pisa-lhc-restarts, ATLAS official blog.

[11] Biondi S., Women of ATLAS - International Woman's Day 2016, OPEN-PHO-LIFE-2016-002.

[12] Biondi S., Alan Litke, inventor of the device that records the neural activity of retinal cells, ATLAS-PHOTO-2016-023. 